\title{
ANALYSIS ON FRACTIONAL FREQUENCY REUSE FACTOR OVER TRADITIONAL FREQUENCY REUSE FACTOR IN CELlular SySTEM
}

\author{
Sharmin Sultana Shompa ${ }^{1}$, Farhana Afroz ${ }^{2}$ and Prof. Dr. A.K.M. Fazlul Haque ${ }^{3}$ \\ ${ }^{1,3}$ Department of Electronics and Telecommunication Engineering, Daffodil International \\ University, Dhaka, Bangladesh \\ ${ }^{2}$ Faculty of Engineering and Information Technology,University of Technology, Sydney, \\ Australia
}

\begin{abstract}
To provide flawless and high speed services among highly increasing users with limited bandwidth has become today's problem. To overcome this problem, a different but efficient frequency allocation system is needed. Instead of conventional Frequency Reuse system, the Fractional Frequency Reuse (FFR) could be a better solution for all voice channels. In this work, a new scheme namely Proposed Fractional Frequency (PFFR) has been proposed as well as a new system model that implements PFFR has been suggested. The main purpose of this analysis is to make a comparative analysis between the performances of PFFR and conventional or Traditional Frequency Reuse Factor (TFRF). Here, the performances of carrier to interference ratio, traffic and trunking efficiency are measured and analyzed to find improvements on frequency planning. In this proposed system module, the whole cellular area has been covered by using both Omni and Sectoring antennas to ensure the full coverage of not only in smaller cells but also at the cell edges of larger cells with the intelligent utilization of PFFR. This analysis is simulated by using a simulation tool MATLAB r2010a. The result of this work has shown a great improvement on CCI with PFFR as compared with the conventional frequency reuse. In this paper it has been shown that the intelligent implementation of PFFR can offer distinct advantages in terms of improved CCI, GoS (Grade of Service), and traffic and trunking efficiency to cellular mobile networks.
\end{abstract}

\section{KEYWORDS}

FFR; PFFR; GoS; Trunking Efficiency; CCI; Omni; Sectoring.

\section{INTRODUCTION}

A flawless communication system requires faster and noiseless connection being maintained all over the area of a cell. With the rapid growth of mobile users, the demand of flawless communication system is increasing day by day. A faster connection can be established by using smart antennas which can cover the large cells as well as smaller cells. Servicing a large number of users with limited bandwidth becomes a great challenge. Reusing frequencies is not the enough solution today. A large number of users are reusing same frequencies repeatedly, which causes the increase of the probability of co-channel interferences. The lower reuse factor for larger cell or higher reuse factor for smaller cell have complex hand-over and high co-channel interference. Since the bandwidth is limited, the frequency allocation should be intelligent. A range of reuse scheme is proposed in [1-8]. Reusing fractional frequency can be done in several ways, such as using Dynamic Fractional Frequency Reuse factors (DFFR) or using dynamic smart antennas. But both are very expensive to implement. If Omni and Sectoring antennas can be used DOI : $10.5121 /$ ijwmn.2015.7506 
efficiently, a system adopting intelligent frequency allocation scheme can be implemented with low cost. By using FFR $(1,4)$ instead of using frequency factor 1, 3, 4, 6, 7 the full cell coverage could be done. So, it would be easy to use fractional frequencies instead of traditional frequency reuse technique.

The Fractional Frequency Reuse can be categorized as- Hard FFR (HFR) and Soft FFR (SFR). The Traditional Frequency Reuse (TFR) is also called HFR which is modified for multi-cell networks. Users of all cell-centres are allocated a common sub-band of frequencies and the rest of the bandwidth is partitioned across cell-edge's users. There is no share of frequencies between cell-edge's users and cell-centre's users, which means there is no cell-to-cell interference or adjacent-cell interferences [2].

On the other hand, the SFR uses partition of the bandwidth in a different way, where the cellcentre users are allowed to share sub-bands with cell-edge users of the other cells. While SFR is more bandwidth efficient than HFR because cell-centre users typically transmit at lower power levels than the cell-edge users which results in more interference to both cell-centre and cell-edge users.

Now considering a Traditional Frequency Reuse Factor (TFRF), if frequency reuse factor 1 is supported, then same frequency will be operated over all cells. This has the capability of maximizing the spectral efficiency but also leads to higher inter-cell interference. Using reuse factor 1, the cell-edge users experience higher degradation as compared with users located nearby base station. This can be reduced if reuse ratio of 4 (classical frequency planning) is considered. Reuse factor 4 means dividing total spectrum band into 4 sub bands and 1 sub band to a given cell has been allocated, so that there would be different frequency bands at the adjacent cells. Meanwhile, in terms of coverage and capacity co-channel interference is reduced at the expense of decrease in efficiency. The main targets of this frequency planning are to enhance channel capacity, optimize spectrum usage, and reduce interference in channels.

For multi-cell systems, if the same frequency resource is reused in each cell of a network, the users at the cell-edge inevitably suffer from the CCI. In this work, a system model of PFFR, which can be an efficient solution to mitigate CCI, has been proposed. Here, to avoid interference at cell edges, a combination of frequency reuse 1 and 4 schemes has been used. The total bandwidth has been divided into two sub bands: one for all cell center users and the other for cell edge users. So, at cell centers of all cells, a frequency reuse 1 sub band is allocated, and for cell edge users a frequency reuse of 4 is allocated. Therefore, full coverage with decreased CCI is maintained by using both Omni and Sectoring antennas. A comparative analysis between the performance of TFRF and PFFR scheme has been made using a simulation tool MATLAB R2010a.

The rest of this paper is organized as follows: In Section 2, the background literature of other authors' works is discussed. It describes the problems and growth of their work on this area. Section 3 concentrates describing the system model which can be a better solution of the problems. Section 4 depicts the simulation process with the simulation tool MATLAB R2010a and analyzes all possible improvements which are graphically represented. Finally the Section 5 concludes the outcome of the work.

\section{BACKGROUND LITERATURE}

An important paper work [1] done by Rizwan Ghaffar, Raymond Knopp and Sophia Antipolice (in 2010) have discussed a combination of the interference suppression for Orthogonal Frequency 
Division Multiple Access (OFDMA) networks and FFR scheme. They have analyzed the celledge users and shown that there is improvement in the spectral efficiency by using FFR scheme. Then the proposed rate allocation and subcarrier ensures interference exploitation by the mobile station (MS). The reduction of power consumption at the base stations (BSs) have found as a result. There only a priori frequency planning is required in the proposed scheme for interference free communication system.

Another paper by Thomas David Novlan, Jeffrey G. Andrews (in 2011) discussed a new analytical framework to evaluate coverage probability and average rate in Strict Fractional Frequency Reuse and Soft Fractional Frequency Reuse (SFFR) systems [2]. They have shown that for actual performance the grid model provides an upper bound. Though their framework was based on the Poisson model, is a lower bound.

Another work by A.K.M Fazlul Haque, A.F.M. Shahen Shah, Md. Abdul Hannan (2011) concentrated on using sectoring antennas instead of Omni antennas for the urban areas [3]. They have shown the improvement on co-channel interference ratio as well as trunking efficiency. Based on simulation, for two propagation environment (urban and rural) two respective models (Sectoring and Omni) are proposed there. Traffic, measurement of the carrier to interference ratio and all factors related with performance has been analyzed here. Finally, it has been experimented in rural and urban areas with Omni and Sectoring antennas and the satisfactory level of traffic has been found.

Kamal Ahmed, Himadri S. Saha, Mustafa M. Hussain, M. R. Amin (in 2011) have done a work [4]. Their purpose was to solve the limitations of WiMax by using three operational band frequencies. Modern technology needs an effective use of the available band for all users. By using several features this problem can be solved. With limited resource this leads to a network to the peak of performance. Maximizing the capacity and upgrading the performance was their main goal and that has been done by using fractional frequency reuse (FFR) with MIMO (MultipleInput Multiple-Output) technique. To overcome the limitations of capacity and coverage, the MIMO is the perfect technique for a WiMax network. At first they have discussed about the features of FFR technology and then to compare the performance of the FFR technology with partial usage of sub-channels (PUSC) technique they have simulated it. To find the best feature for Wimax network on real RF environment the simulation results are compared, discussed and concluded with some future work.

An important contribution of this work has given by Chandra Thapa and Chandrasekhar (in 2012). In their work, they have proposed a mix frequency reuse FFR $(1,3)$ to avoid interference at cell edges. In this reuse scheme, the total bandwidth is divided into two sub bands: for all cell center users a frequency reuse 1 sub band is allocated, and for all cell edge users a frequency reuse of 3 is allocated [5]. The overall comparative evaluation of FFR and classical frequency reuse for downlink homogenous condition in 3GPP-LTE has been shown at their work. Results show that FFR $(1,3)$ can reduce the interference effectively and balances the resources utilization. This also shows that it provides better probability of acceptance rate and probability of coverage than the classical one.

In [6], Qian Li (2013) described about Heterogeneous Wireless Networks where using optimal FFR and power control scheme to coordinate the interference among high power and low power nodes. The scheme can be optimized to maximize the sum of the long term log-scale throughput. The Lagrange dual function is first derived for the proposed optimization problem at their work. To search the optimal solution, the gradient descent method has been used for the convex dual problem. The optimal solution for the dual problem is also the optimal solution for the primal 
problem. Finally, their simulation results show that the proposed scheme can greatly improve the performance on system capacity and user experience in the wireless heterogeneous network.

In [7], Cheng Chen, Nikola and Harald (2013) worked on optical wireless cellular network using fractional frequencies. They have shown the user performance and throughput of cell edge and cell center areas. Also they have proved that using Light Emitting Diode (LED) for transmission of optical power can satisfy the requirements of the illumination.

A different work in [8] was done by Christos Bouras and Vasileios Kikkoinos (2013) who have concentrated on using integrated femtocell and macrocell with Fractional Frequency to overcome CCI. They have proposed a method and applied it in an integrated femtocel/macrocell environment precisely. Finally based on the optimization of three metrics it's been evaluated depending on the network operator's needs.

Another related work [9] done by Giovanni Giambene and Van Anh Le (2014) also discussed about using the other types of FFR for $4 \mathrm{G}$ Long Term Evoluation (LTE) system. To achieve the higher capacity under some fairness constraints they have concentrated on identifying the FFR scheme in such a way where to maximize cell capacity in different conditions by means of Ns-3 simulations it can perform an exhaustive-search optimization of the selected FFR scheme. To gain higher capacity they have distinguished between two FFR schemes where different reuse patterns and different transmission patterns are adopted.

\section{PFFR SCHEME ANd The System Model OF PFFR}

In this section, the PFFR scheme and the system model using PFFR have been described. PFFR scheme is basically the utilization of fractional frequency reuse factor $(1,4)$ where the bandwidth is partitioned or fractioned in 5 parts (sub-bands) for cell coverage. Then a system model using PFFR is designed by using both Omni and tri-sectoring antennas. Omni directional antennas can cover the cell horizontally (as azimuth) and the tri-sectoring directional $120^{\circ}$ antennas can cover the cell in three directions with maximum radio frequency [4]. Both scheme and system model, which are aimed to improve CCI, are illustrated. In this system model of PFFR, the following steps are required-

a) Cell splitting

b) Both Omni directional and tri-sectoring (120 degree) antennas

c) $(1,4)$ FFR planning

At first the cell needs to be splitted with $3 \mathrm{~km}$ by $3 \mathrm{~km}$ square centered on base stations. After that, the whole bandwidth (BW) is fractioned into 5 parts. Frequency reuse factor 1 has been used for cell center and the other reuse factor 4 has been used for cell edges.

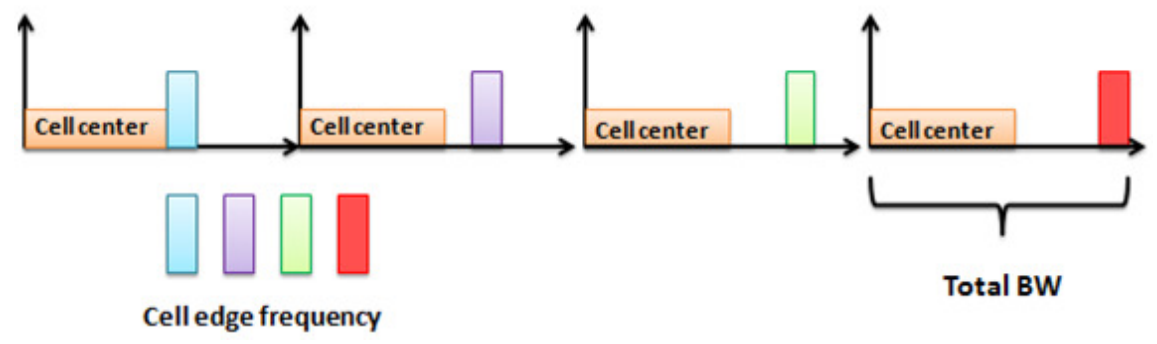

Figure 1. Bandwidth utilization using fractional frequency reuse factor. 
Then the whole cellular system structured as a combined form of both Omni FFR and sectoring FFR as shown in the following figure.

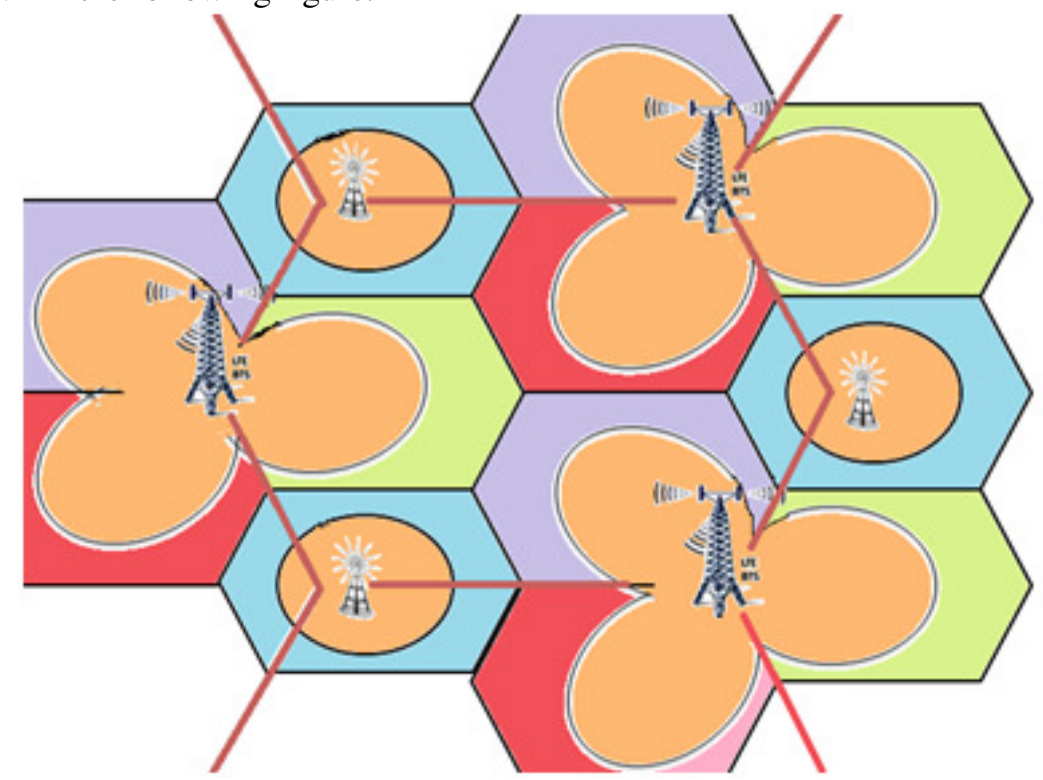

Figure 2. Structural diagram of PFFR system model.

\subsection{Advantages of Proposed FFR system}

Multi channel frequency distribution over distributed network will cause factor reduction. Fractional frequency reuse (FFR) has been proposed as an ICIC technique in OFDMA based wireless networks [2]. The basic idea of FFR is to partition the total bandwidth in such a way where the cell-centre users and cell-edge users of the network can deal with the adjacent cell interferences. It could be done to achieve three major conditions: (i) cell-edge users of adjacent cells do not interfere with each other and (ii) co-channel interferences among cells can be reduced, while (iii) using more total spectrum than conventional frequency reuse. The use of FFR can show the improvement in rate and sum network throughput and spectral efficiency and coverage for cell-edge users.

Sectorization with fractional frequency reuse technique is beneficial in cellular systems which can reduce CCI. Because of the reduced interference, a more efficient frequency reuse plan can be allowed by sectored cellular systems with the advantages of Omni antennas. At the following figure the advantage of proposed system model of PFFR has been shown. Here, Cell A and cell B different frequency resources $\mathrm{f} 3$ and $\mathrm{f} 2$ are allocated to $\mathrm{A} 1$ and $\mathrm{B} 1$ at the cell edge. This allows enhancing the communication quality with no interference [10]. Cell B allocates f3 to a UE that uses low transmitting power. Now, if cell A is already using f3 which uses high transmitting power for a UE at the cell edge then no interference occurs at their cell edges. 


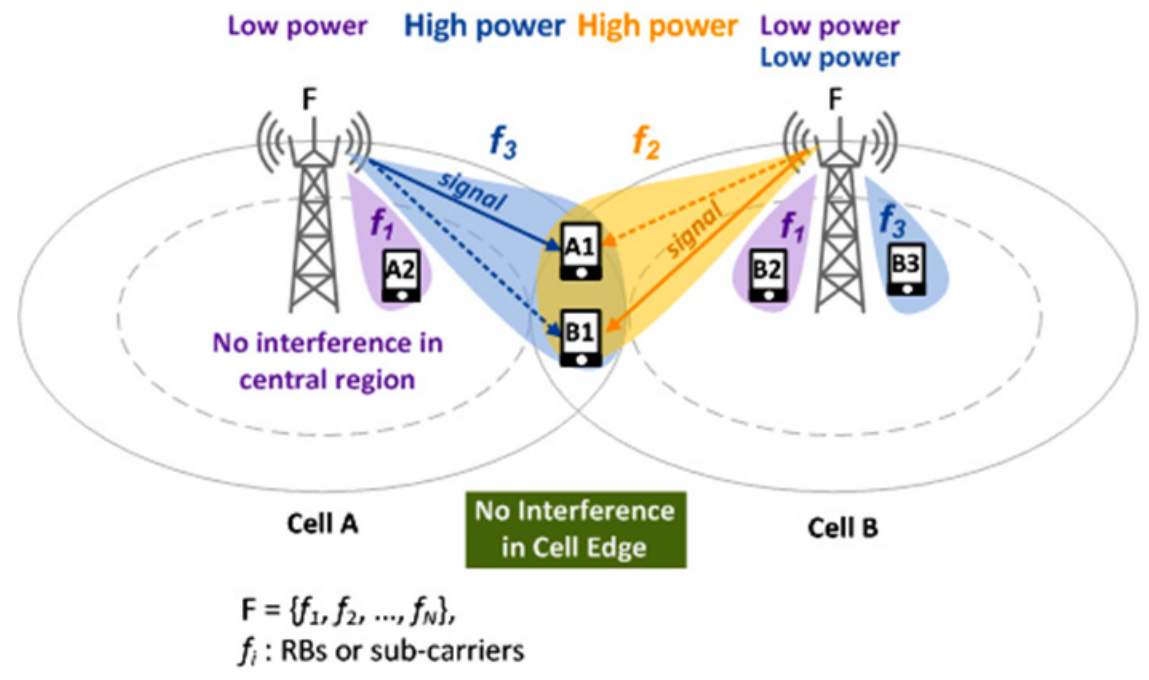

Figure 3. Advantages of using FFR for both cell centers and cell edges.

The solution of this interference problem is the Proposed Fractional Frequency Reuse (FFR). In FFR, a fraction of all sub-channels are available for the users at the cell/sector edge while the cell-centre users operate with all sub-channels available [1]. By eliminating inter-cell interference this PFFR and its proposed system model can improve reliability of cellular networks while their capacity gains are only marginal.

\subsection{Proposed FFR Requirements}

In this subsection, the requirements of proposed FFR are reported as follows.

Assuming the total bandwidth75 MHz for GSM 1800 servicing 512 channels $3 \mathrm{~km}$ by $3 \mathrm{~km}$ square area cell splitting 1200 sectoring and Omni antennas

The factors, which have been considered for performance analysis, are Channel Capacity or Traffic, Trunking efficiency, Grade of Service (GoS), and Carrier to Interference ratio or CoChannel Interference (C/I or CCI). Grade of Service $(\mathrm{GoS})$ is defined as the probability of failure of call [3]. The available voice channel per cell is called Channel Capacity. Traffic is measured by Erlangs. In telecommunication networks, during a specified period of time (normally a busy hour) the traffic intensity is a measure of the average occupancy of a server [10]. Though recently an optimal fractional frequency reuse with the power control scheme has been proposed, it would be expensive to install everywhere. This scheme coordinates the interference among high power and low power nodes which can be optimized to maximize the sum of the long term log-scale throughput among all the user equipments (UEs) [6].

Channel Capacity $=($ No. of available voice channel $) /($ Frequency reuse factor $)$

Erlangs $=($ No. of calls $) /($ average call holding time $)$

The relation between the $\mathrm{CCI}$ and $\mathrm{C} / \mathrm{I}$ is $\mathrm{CCI}(\mathrm{dB})=-\mathrm{C} / \mathrm{I}(\mathrm{dB})$. So, the carrier to interference ratio is given by

$\mathrm{C} / \mathrm{I}=10 \log \left[1 / \mathrm{j} *(\mathrm{D} / \mathrm{R})^{\gamma}\right]$ 
Where,

$\mathrm{j}=$ number of co-channel interferer

$\gamma=$ propagation constant

$\mathrm{D}=$ frequency reuse distance

$\mathrm{R}=$ radius of the cell

The above equation may be written as

$$
\mathrm{C} / \mathrm{I}=10 \log \left[1 / \mathrm{j} *\left\{\sqrt{ }(3 \mathrm{~K})^{\gamma}\right\}\right]
$$

Where,

$\mathrm{D} / \mathrm{R}=\sqrt{ }(3 \mathrm{~K}) ; \mathrm{K}=$ frequency reuse factor

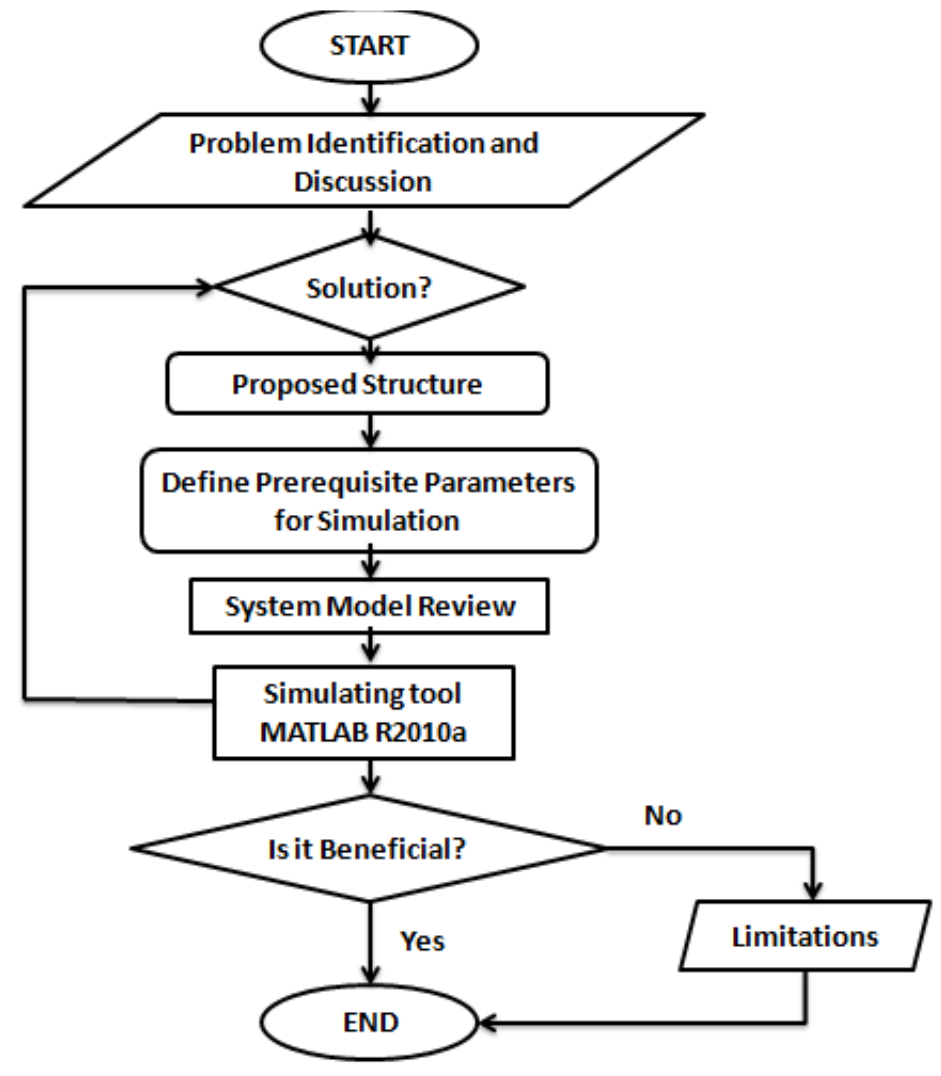

Figure 4.Flow chart.

Considering PFFR and the proposed system model of PFFR, the method of FFR $(1,4)$ would be the main priority. If this model simulated successfully then some results with advantages and limitations and also the findings may come from it, else the solution would be under reconsideration until a better progress has done. 


\section{Simulation AND ANALYSIS}

The analysis has been done by using MATLABR2010a for various frequency reuse factors and fractional frequency reuse for both Sectoring and OMNI cell for $2 \%$ GoS (urban). The simulated values for traditional frequency reuse factors are shown in Table 1 for OMNI cell and Table 2 for Sectoring cell. Table 3 has shown the performance of proposed fractional frequency reuse which is also described in Figure 5 to figure 8. All measurement has been calculated by using equation 3,4 and 5 .

Table 1. Performance evolution of Traditional Frequency Reuse Factors (TFRF) for Omni Cell

\begin{tabular}{|c|c|c|c|c|}
\hline $\begin{array}{c}\text { TFRF } \\
(\text { Omni })\end{array}$ & $\begin{array}{c}\text { No. of } \\
\text { channel per } \\
\text { cell }\end{array}$ & $\begin{array}{c}\text { Traffic with 2\% } \\
\text { GOS (Erlang) }\end{array}$ & $\begin{array}{c}\text { Trunking Efficiency } \\
(2 \% \text { GOS })\end{array}$ & C/I (DB) \\
\hline 1 & 512 & 506.4 & $99 \%$ & 1.76 \\
\hline 3 & 171 & 157.5 & $92 \%$ & 11.3 \\
\hline 4 & 128 & 115.2 & $90 \%$ & 13.8 \\
\hline 7 & 73 & 62 & $85 \%$ & 18.7 \\
\hline
\end{tabular}

Table 2. Performance evolution of Traditional Frequency Reuse Factors (TFRF) for Sectoring Cell

\begin{tabular}{|c|c|c|c|c|}
\hline $\begin{array}{c}\text { TFRF } \\
\text { (Sector 3) }\end{array}$ & $\begin{array}{c}\text { No. of } \\
\text { channel per } \\
\text { cell }\end{array}$ & $\begin{array}{c}\text { Traffic with 2\% } \\
\text { GOS (Erlangs) }\end{array}$ & $\begin{array}{c}\text { Trunking Efficiency } \\
(2 \% \text { GOS) }\end{array}$ & C/I (DB) \\
\hline 1 & 171 & 157.5 & $92 \%$ & 6.53 \\
\hline 3 & 57 & 46.8 & $82 \%$ & 16 \\
\hline 4 & 43 & 34 & $79 \%$ & 18.6 \\
\hline 7 & 25 & 17.5 & $68 \%$ & 23.4 \\
\hline
\end{tabular}

Table 3. Performance evolution of Fractional frequency reuse (PFFR) for Both Omni and Sectoring Cell

\begin{tabular}{|c|c|c|c|c|c|}
\hline PFFR & Antenna & $\begin{array}{c}\text { No. of channel } \\
\text { per cell }\end{array}$ & $\begin{array}{c}\text { Traffic with } \\
2 \% \text { GOS } \\
\text { (Erlangs) }\end{array}$ & $\begin{array}{c}\text { Trunking } \\
\text { Efficiency }(2 \% \\
\text { GOS) }\end{array}$ & C/I (DB) \\
\hline 1 & 3 sector & 171 & 157.5 & $92 \%$ & 6.53 \\
\hline 4 & 3 sector & 43 & 34 & $79 \%$ & 18.6 \\
\hline \multicolumn{5}{|l}{} \\
\hline 1 & Omni & 512 & 506.4 & $99 \%$ & 1.76 \\
\hline 4 & Omni & 128 & 115.2 & $90 \%$ & 13.8 \\
\hline
\end{tabular}

\subsection{Results and Findings}

In this subsection, the simulation results and findings from the analysis are discussed sequentially. Channel Capacity will give the results on depict frequency levels, instead of multiple distribution levels.

In Fig. 5 CCI vs. TFRF and PFFR graph is depicted. It is observed from the figure that the trunking efficiency depends on Traffic and Traffic depends on number of channels and finally 
number of channel depends on frequency reuse factors. The CCI for TFRF and PFFR is measured with Omni and Sectoring antennas. It is obvious that with the increase of using fractional frequency reuse factor, the co-channel interference decreases. Since trunking efficiency depends on Traffic in Erlangs, it is clear that, for using FFR Trunking efficiency decreases.

In Fig. 6, the performance of traffic for GoS (Grade of Service) 2\% versus PFFR and TFRF for Omni and Sectoring antennas has shown. For a good channel utilization blocking rate $2 \%$ needs better tunking efficiency which is provided by PFFR perfectly. TFRF has very poor performance as compared with PFFR in both Omni antennas and sectoring antennas. Here PFFR shows better performance compared with TFRF in terms of traffic for GoS $2 \%$.

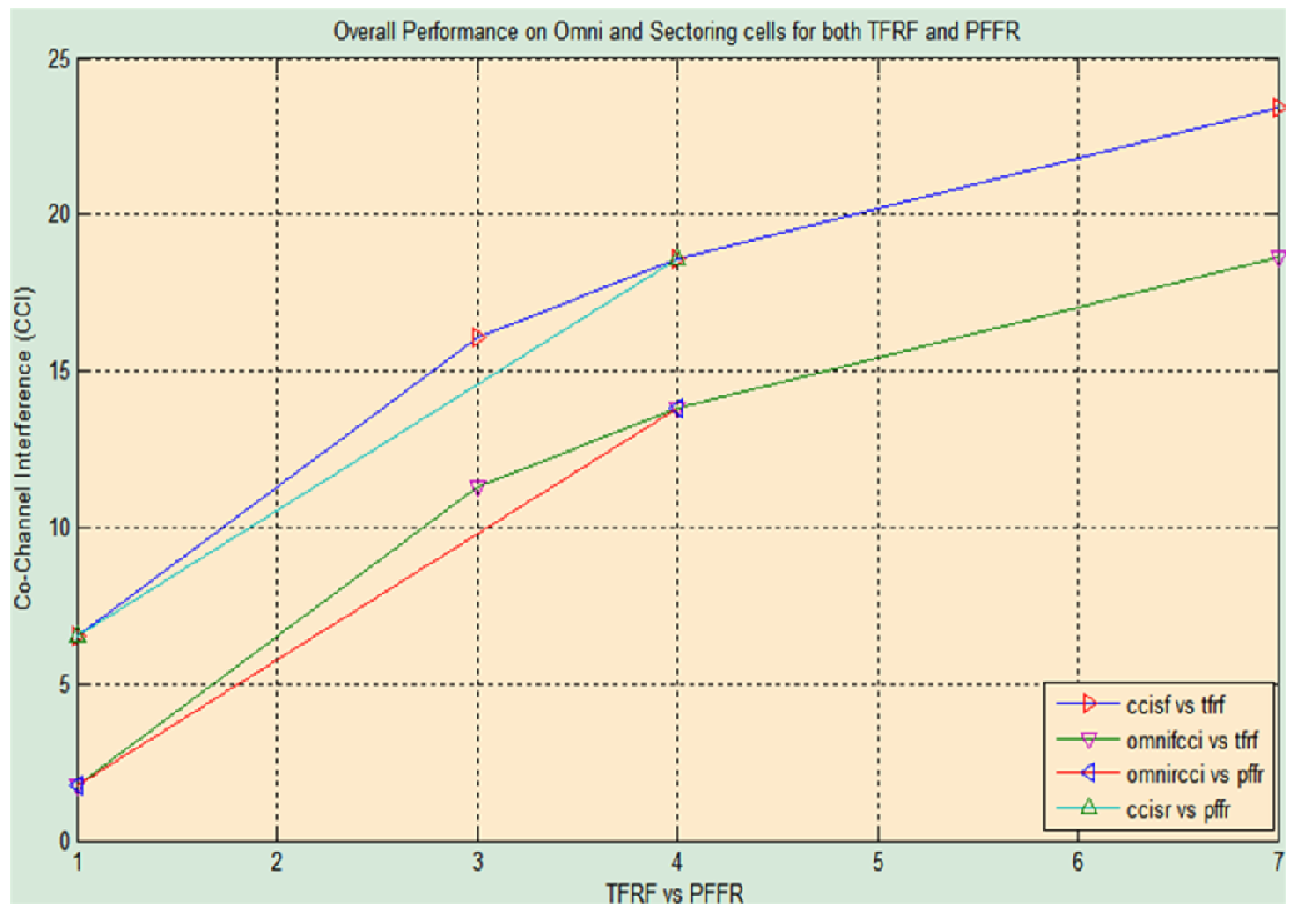

Figure 5. Graphical representation of the comparative performance on Co-channel-interference ratio of Traditional Frequency Reuse Factor (TFRF) and Proposed Fractional Frequency Reuse Factor (PFFR) for both Omni and sectoring cells. 


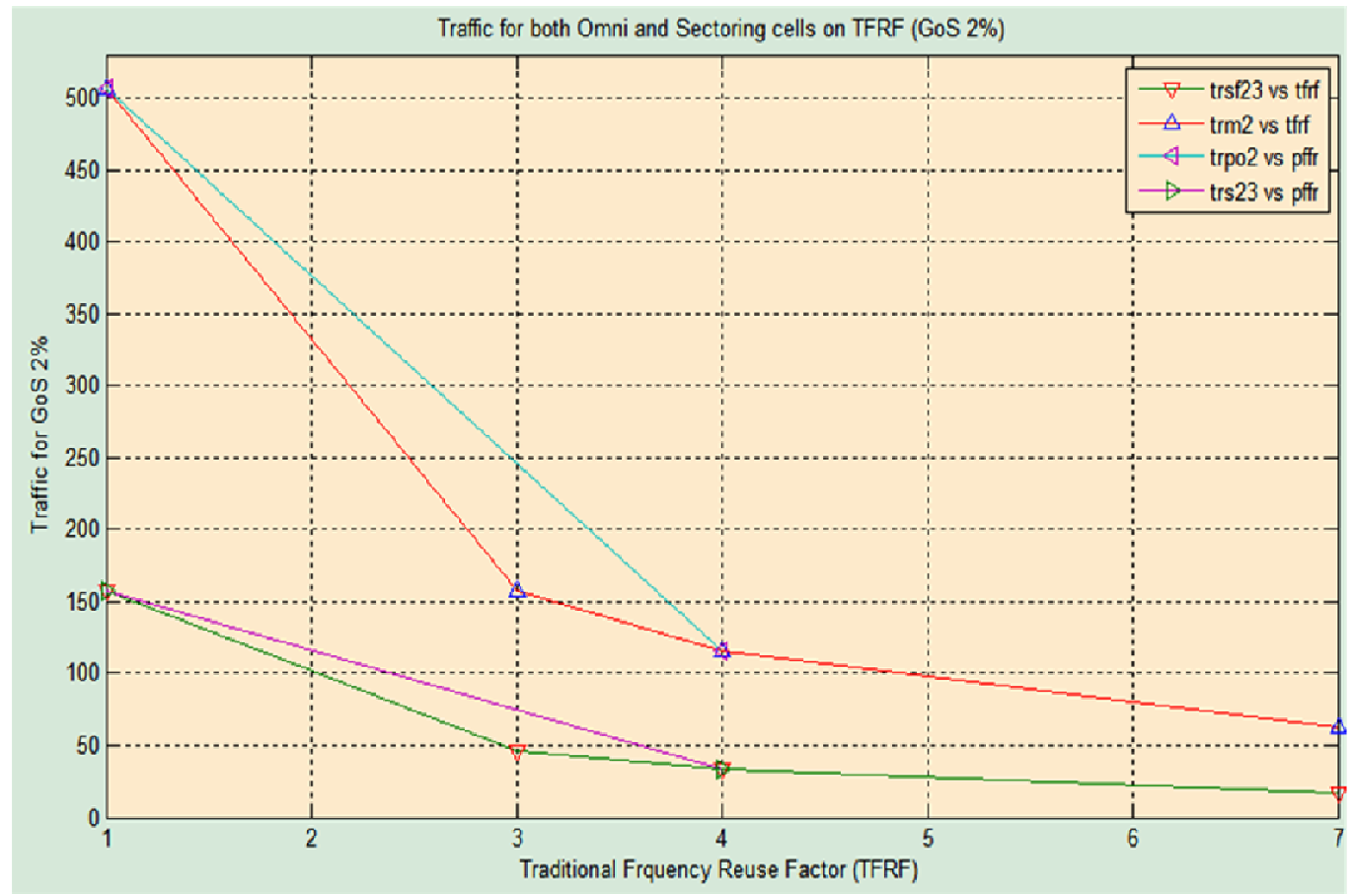

Figure 6. Traffic (for $2 \% \mathrm{GoS}$ ) for both Omni and sectoring cells on TFRF and PFFR.

Figure 7 and 8 shows the relation between TFRF and PFFR for both OMNI and Sectoring cell from where one can easily take the decision about which scheme performs better in OMNI and Sectoring cell concept.

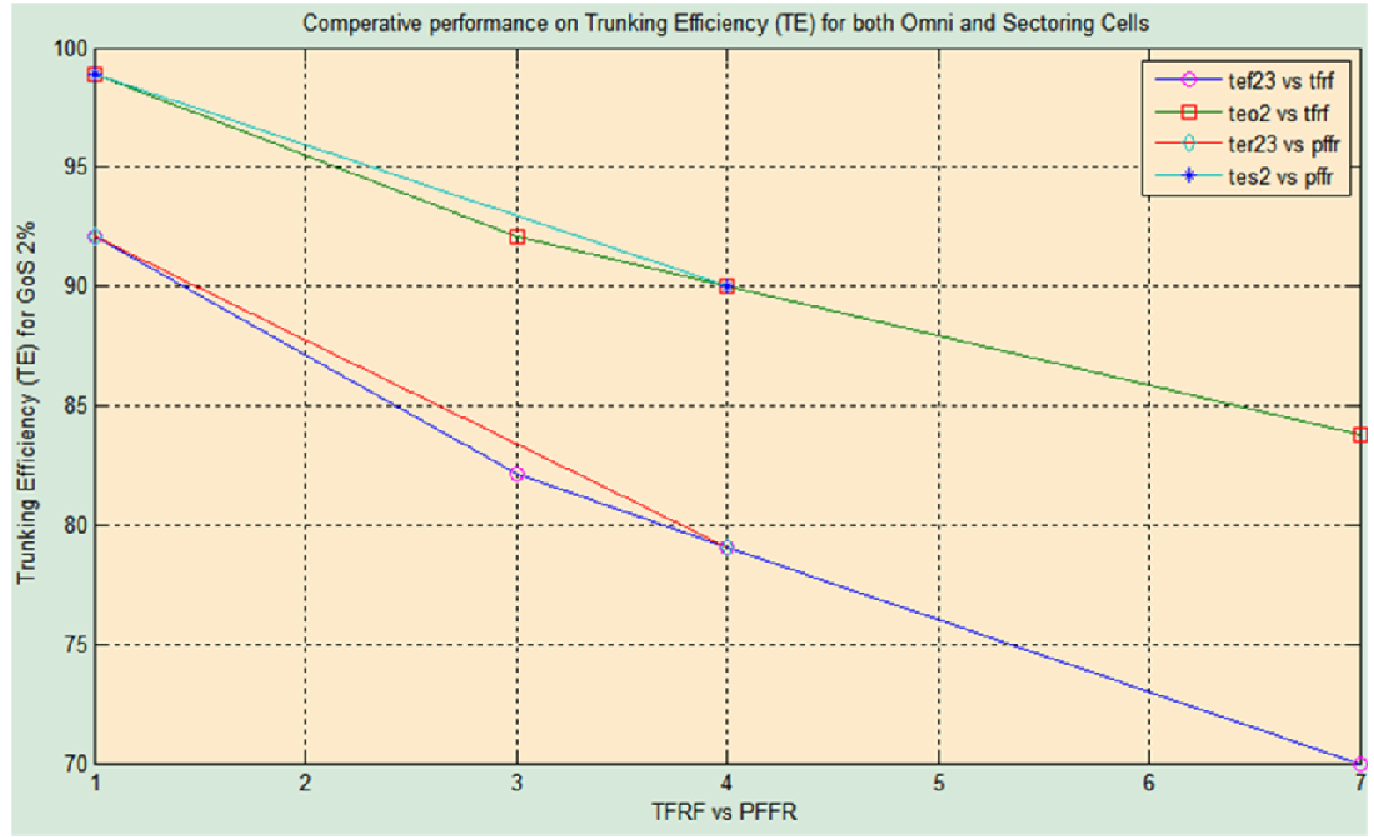

Figure 7. Comparative performance on trunking efficiency for both Omni and sectoring cells on TFRF and PFFR. 
Fig. 7 shows the comparative performance of trunking efficiency for both Omni and sectoring cells on TFRF and PFFR. Here PFFR shows better performance compared with TFRF in terms of trunking efficiency. This graph is representing the channel capacity on $2 \% \mathrm{GoS}$.

In Fig. 8, the main comparison between TFRF and PFFR scheme with system model is depicted. There is noticeable CCI for TFRF. But the PFFR with system model is giving much more improvement on CCI. It is to be noted that Traffic not only depends on number of channel but also depends on GoS.

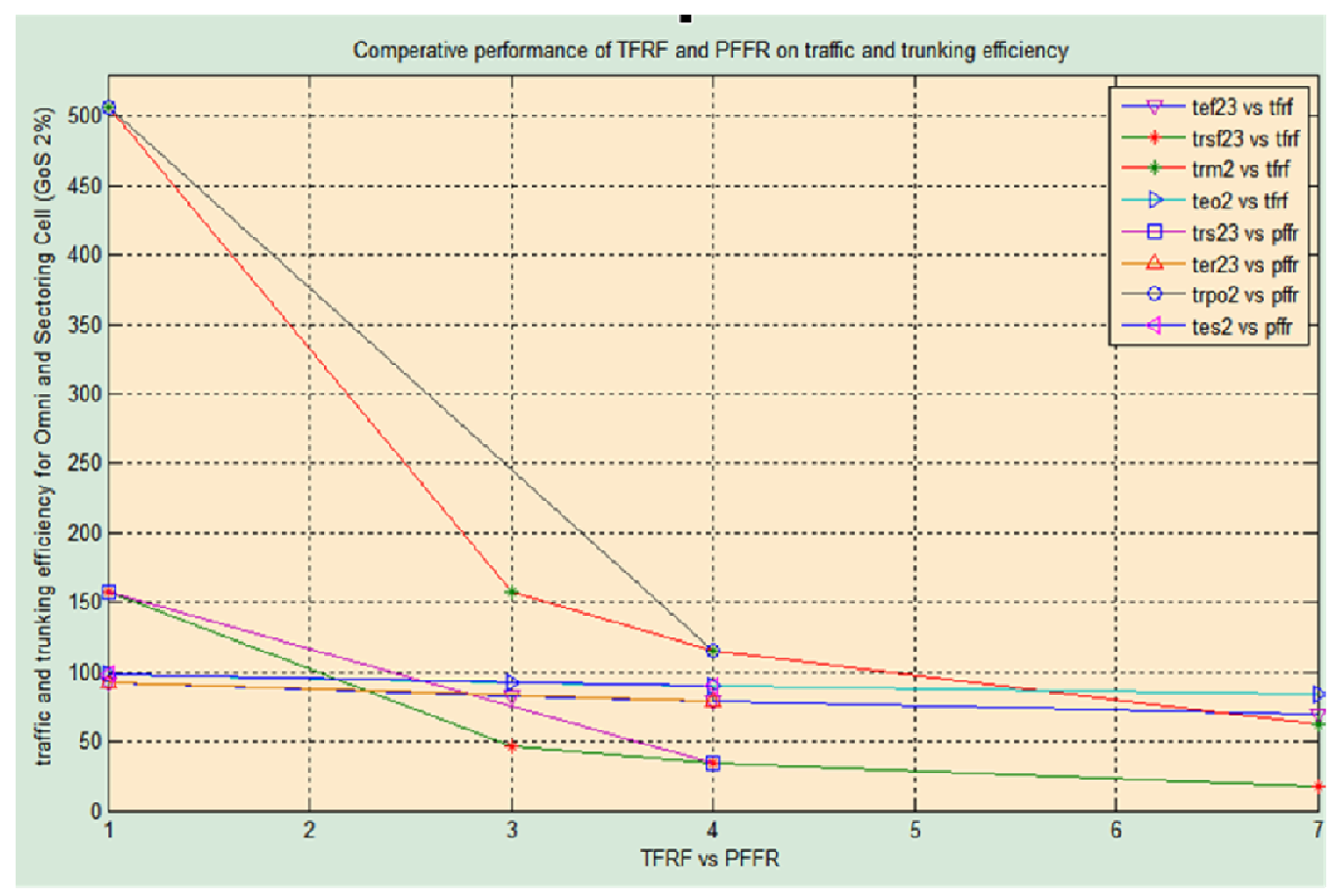

Figure 8. Comparative performance on Traffic and Trunking Efficiency (GoS 2\%) for both Omni and sectoring cells on TFRF and PFFR.

The key findings are that PFFR provides considerably higher coverage than a Traditional Reuse 1, 3, 4 or 7 Factors, with the PFFR coverage being comparable to that of a Reuse 4 factor at the cell edges and factor 1 at the cell centers. Additionally, PFFR and the system model of PFFR have shown a significant improvement in C/I ratio compared with a TFRF.

\section{CONCLuSiON}

In this analysis, the performance of frequency reuse schemes in mobile cellular environment has been simulated and evaluated. This has been evacuated in two types of frequency schemes- TFRF and PFFR. Based on simulation, for $2 \%$ GoS two respective models of propagation environment (Sectoring and Omni cells) have been proposed. The co-channel interference ratios with demand of the traffic and trunking efficiency which are related with performance are simulated carefully. In this paper, a fractional frequency reuse scheme namely PFFR has been proposed as well as a system model employing this scheme has been suggested. Furthermore, a comparison between the performances of TFRF and PFFR is analysed based on trunking efficiency and CCI 
with considering urban areas. PFFR with proposed system model has been found the satisfactory level. Intelligent use of fractional frequency reuse factors is proven better performance rather than the usual reuse factor scheme. The only limitation of system model of PFFR is that the data rate may not be increased as well as the widow size remains the same. The result of this work has shown a great improvement on CCI with FFR as compared with the conventional frequency reuse. Sectorlization with fractional frequency technique will give results in single cellur networks, but not more for distributed network. The future work of this paper includes studying the performance of PFFR while it is implemented in smart antenna array system. The cost of installing smart antenna would be high, but using smart antenna can reduce CCI more significantly.

\section{REFERENCES}

[1] Rizwan Ghaffar, Raymond Knopp, "Fractional Frequency Reuse and Interference Suppression for OFDMA Networks”, Eurecom's research, 2011.

[2] Thomas David Novlan, Radha Krishna Ganti, Arunabha Ghosh, Jeffrey G. Andrews, "Analytical Evaluation of Fractional Frequency Reuse for OFDMA Cellular Networks", IEEE Transaction on wireless communicaitons, Vol. 10, No. 12, pp.4294-4305,December 2011.

[3] A.K.M Fazlul Haque, A.F.M. Shahen Shah, Md. Abdul Hannan, Nusrat Jahan, jasim Uddin Ahmed, Md. Abu Saleh "High Performance Analysis of Frequency Reuse Schemes in Cellular Mobile Environment”, (IJLTC) (E-ISSN: 2045-5364) Volume 2, Issue 1, March 2011.

[4] Kamal Ahmed, Himadri S. Saha, Mustafa M. Hussain, M. R. Amin, "Comparison between the performance of PUSC and FFR network "IJSCE ISSN: 2231-2307, Volume-1, Issue-5, November 2011.

[5] Chandra Thapa and Chandrasekhar, "Comparative evaluation of Fractional Frequency Reuse (FFR) and Traditional Frequency Reuse in 3gpp-lte downlink”, (IJMNCT) Vol.2, No.4, August 2012.

[6] Qian Li "Optimal Fractional Frequency Reuse and Power Control in the Heterogeneous Wireless Networks" Wireless Communications, IEEE Transactions on (Volume:12, Issue: 6 ) 2013.

[7] Cheng Chen, Nikola Serafimovski and Harald Haas "Fractional Frequency Reuse in Optical Wireless Cellular Networks" 2013 IEEE 24th International Symposium on Personal, Indoor and Mobile Radio Communications: Special Sessions.

[8] Christos Bouras, Vasileios Kikkoinos "Fractional Frequency Reuse in Integrated Femtocell/ Macrocell Environment" WWIC 2013 Springer-Verlag Berlin Heidelberg sumission.

[9] Giovanni Giambene, Van Anh Le "Performance Evaluation of Different Fractional Frequency Reuse Schemes for LTE" GTTI 2014 Thematic Area.

[10] https:// en.wikipedia.org/wiki/traffic_intensity

[11] T.Bonald, S.C. Borst, and A. Proutiere, "Inter-cell scheduling in wireless data networks", in Proceeding of European wireless conference, 2005.

[12] K.doppler, c.Wijting and K. Valkealahti, "Interference aware scheduling for soft frequency reuse", in Proc. IEEE Vehicular Technology Conf., Barcelona, April 2009, pp.1-5.

[13 ]Zerihun Abate, "WiMax RF Systems Engineering” 2009: 250, third edition. 


\section{AUTHORS}

SHARMIN SULTANA SHOMPA completed her M.Sc. Engineering degree in Electronics and Telecommunication Engineering from Daffodil International University, Dhaka, Bangladesh and B. Sc. Engineering in Electronics and Telecommunication Engineering from University of Liberal Arts Bangladesh. She is currently working as a Lecturer of Electronics and Communication Engineering department, Bangladesh Institute of Science and Technology (BIST), Dhaka, Bangladesh.

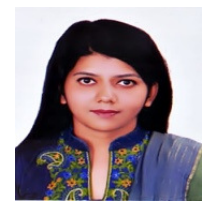

FARHANA AFROZ obtained her MEStud degree with Telecommunication Engineering maj or from University of Technology, Sydney, Australia. She completed MS and B.Sc. (Honours) degree from the department of Applied Physics, Electronics and Communication Engineering of University of Dhaka, Bangladesh. Her current research interest includes $4 \mathrm{G}$ mobile networks and digital signal processing.

PROF. DR. A.K.M. FAZLUL HAQUE is the Department Head of Electronics and Telecommunication Engineering at Daffodil International University Dhaka, Bangladesh.

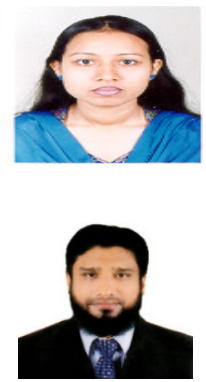

